\title{
ESTIMATING CRITICAL PATH AND ARC PROBABILITIES IN STOCHASTIC ACTIVITY NETWORR'S
}

\author{
George S. Fishman \\ Technical Report No. UNC/ORSA/TR-83/5 \\ Auqust 1983
}

Curriculum in Operations Research and Systems Analysis

University of North Carolina at Chapel Hill
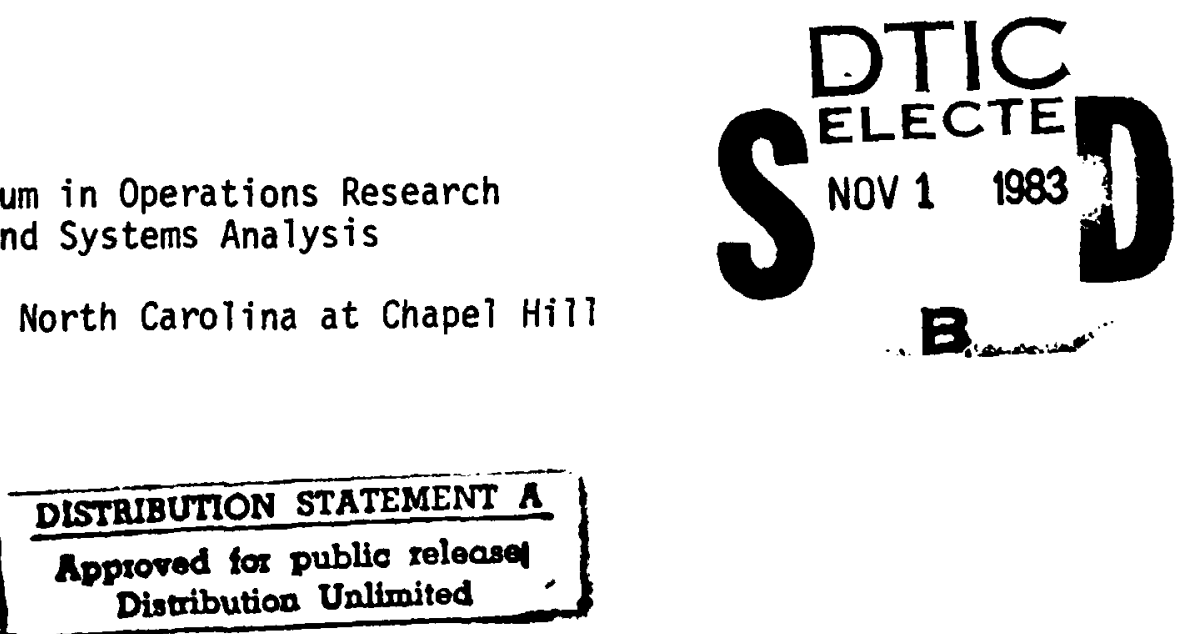

This research was supported by the Office of Naval Research under contract N00014-76-C-0302. Reproduction in whole or part is permitted for any purpose of the United States government. 
I am grateful to Professor Veena Adlakha for her helpful comments on this paper.

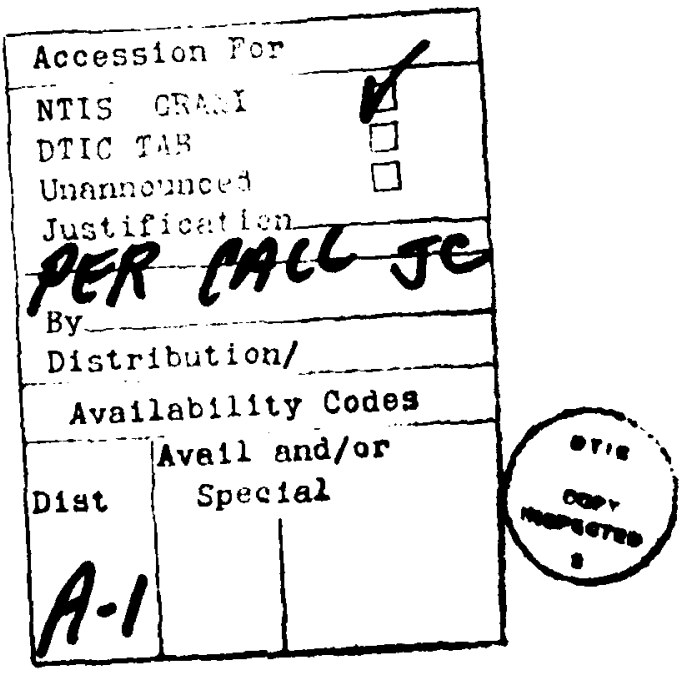




\section{Introduction}

Although comprehensive procedures have been developed for analyzing an activity network with deterministic arc completion times (e.g., Elmaghraby 1977), no comparable set of comprehensive procedures exists for a network in which the arc completion times are random. In particular, a stochastic activity network is properly characterized by distributional information. For example, on independent passes through such a network from source to sink, the network completion times on each pass usually differ, as do the paths identified as the critical paths. Here, the distribution function (d.f.) of network completion time and the frequency with which each path is the critical path provide useful characterizations for describing network behavior.

Whereas conventional search algorithms have been the principal tools of analysis for the deterministic case, Monte Carlo methods have been the principal tool for the stochastic case. For example, see Elmaghraby (1977) and Sullivan, Hayya and Schaul (1982). However, the performance of the Monte Carlo method has not been exceptional until recently when Sigal, Pritsker and Solberg (1979) showed how one could use information on a cutset of a network to improve the accuracy of estimate for a given cost. More recently, Fishman (1983) showed how the cutset information can be used together with quasirandom points and series reduction to estimate the d.fs. of network completion time and shortest path time with upper bounds on the errors of estimate that converge more rapidly than $0\left(1 / k^{1 / 2}\right)$, the rate for the standard error in a Monte Carlo experiment using random sampling on $K$ independent replications.

The purpose of this paper is to extend this combined cutset/quasirandom points/series reduction approach to the estimation of the probability that path $m$ is the longest path, the probability that path $m$ is the shortest 
path, the probability that arc $i$ is on the longest path and the probability that arc $i$ is on the shortest path. Section 1 introduces the necessary notation. Section 2 describes the use of cutset information, as proposed by Sigal, Pritsker and Solberg (1979), to estimate a parameter by Monte Carlo methods with less variance than would obtain for the same number of replications when using crude Monte Carlo techniques. Section 3 then describes how one can use quasirandom points with the cutset information on each replication to make the error of estimate converge more rapidly than in the case of random sampling. Section 4 extends the analysis to the remaining estimators of interest. Section 5 describes how series reduction in the network can work to advantage by reducing the number of dimensions sampled. Section 6 then describes an experimental design for evaluating the benefits to be derived from using cutset information together with quasirandom points and series reduction. Section 7 presents an example which assesses the benefits of each sampling plan versus its relative computation time.

1. Definitions

Consider an acyclic directed network with a single source, single sink, $N$ arcs and $L$ paths. Let $x_{1}, \ldots, x_{N}$, the passage times for arcs $1, \ldots, N$, be independent random variables where $x_{i}$ has distribution function (d.f.) $F_{i}$ on $[0, \infty)$ and inverse distribution function $G_{j}(u)=\min \left[x: F_{j}(x) \geq u\right.$, $0 \leq u<1]$. The completion time of path $m$ is

$$
T_{m}=\sum_{i=1}^{N} a_{i m} x_{i}=\sum_{i \in I_{m}} x_{i}
$$

where $a_{i m}=1$ if arc $i$ is on path $m, a_{i m}=\dot{u}$ otherwise and $I_{m}$ denotes the set of arcs on path $m$. Consider the network completion time

$$
T^{\star}=\max \left(T_{1}, \ldots, T_{L}\right)
$$


and the shortest path time

$$
T_{\star}=\min \left(T_{1}, \ldots, T_{L}\right)
$$

Characterizations of the network include:
a. $\operatorname{pr}\left(T^{\star} \leq t\right)$
$0 \leq t<\infty$
b. $E T^{\star}$
c. $g_{m}=p r(m$ is the longest path)
$m=1, \ldots, L$
d. $r_{i}=p r$ (arc $i$ is on the longest path) $i=1, \ldots, N$
e. $\operatorname{pr}\left(T_{*} \leq t\right)$
$0 \leq t<\infty$
f. $E T_{\star}$
g. $h_{m}=\operatorname{pr}(m$ is the shortest path) $m=1, \ldots, L$
h. $s_{i}=\operatorname{pr}(\operatorname{arc} i$ is on the shortest path $) \quad i=1, \ldots, N$.

Fishman (1983) describes a highly efficient method of estimating $a, b$, e and $f$ based on cutset information, quasirandom points, and series reduction. The present paper extends the method to the estimation of the probabilities $\left\{g_{m} ; m=1, \ldots, L\right\},\left\{h_{m} ; m=1, \ldots, L\right\},\left\{r_{i} ; i=1, \ldots, N\right\}$ and $\left\{s_{i} ; i=1, \ldots, N\right\}$.

2. Cutsets

Observe that

$$
g_{m}=\operatorname{pr}\left[T_{m}>T_{n} ; n \neq m, n=1, \ldots, L\right] \quad m=1, \ldots, L \text {. }
$$

Because of the shared arcs among paths, $T_{1}, \ldots, T_{L}$ are usually not independent and therefore $g_{m}$ generally has no readily accessible analytical form. However, one can make use of the cutset information as in Sigai, Pritsker and Solberg (1979) to derive a useful expression for estimating $a_{m}$.

Let $H$ denote a cutset of the network. As used here, a cutset denotes a set of arcs that connects a set of nodes $w$ containing the source with 
a set of nodes $\bar{w}$ containing the sink. Also assume that each path has only one arc in $H$. If each arc in $H$ points from $\omega$ to $\bar{\omega}, H$ is called a uniformly directed cutset (UDC). We assume that $H$ is a UDC. Let

$$
S_{m}=T_{m}-X_{i_{m}} \quad m=1, \ldots, L
$$

and

$$
\gamma_{i}=\sup _{\substack{m=1, \ldots, L \\ a_{i m}=1}} S_{m} \quad i \in H
$$

where $i_{m}=I_{m} \cap H$ denotes the arc on path $m$ that is in the cutset.

Then $x_{j}+Y_{i}$ is the maximal time of all paths that use arc $i \quad i \in H$ and $\operatorname{pr}\left(m\right.$ is the longest $\left.\operatorname{path} \mid S_{1}, \ldots, S_{L}\right)$

$$
\begin{aligned}
& \left.=0 \int_{\substack{i \in H \\
i \neq i_{m}}}^{\infty} F_{i}\left(t-Y_{i}\right)\right] d F_{i_{m}}\left(t-S_{m}\right) \text { if } Y_{i_{m}}=S_{m} \\
& =0 \quad \text { otherwise. }
\end{aligned}
$$

Therefore,

$$
\begin{array}{rlrl}
A_{m} & =\pi_{\substack{i \in H \\
i \neq i_{m}}} F_{i}\left(T_{m}-Y_{i}\right) & \text { if } \quad T_{m}=Y_{i_{m}}+X_{i} \\
& =0 & & \text { otherwise }
\end{array}
$$

has expectation $g_{m}$. Let the subscript $j$ denote replication $j$. Then for $K$ replications

$$
\hat{g}_{m K}=\frac{1}{K} \sum_{j=1}^{K} \delta\left(T_{m j}-Y_{i_{m} j}-x_{i} j\right) \pi_{\substack{i \in H \\ i \neq i_{m}}} F_{i}\left(T_{m j}-Y_{i j}\right),
$$

where

$$
\begin{aligned}
\delta(x) & =1 & & \text { if } x=0 \\
& =0 & & \text { otherwise, }
\end{aligned}
$$


is an unbiased estimator of $g_{m}$. If the replications are statistically independent, then var $\hat{g}_{\mathrm{mK}}$ is proportional to $1 / \mathrm{K}$.

In principle, (8) is the estimator of $g_{m}$ that Sigal, Pritsker and Solberg propose. They note that it should give smaller variance for $K$ independent replications than the more conventional estimator

$$
\tilde{g}_{m K}=\frac{1}{K} \sum_{j=1}^{K} \delta\left(T_{j}^{*}-T_{m j}\right)
$$

where

$$
T_{j}^{*}=\max \left(T_{1 j}, \ldots, T_{L j}\right) .
$$

However, both var $\hat{g}_{m K}$ and $\operatorname{var} \tilde{g}_{m K}$ converge as $1 / K$. In this paper we propose the use of quasirandom points to speed up the convergence of the error of estimate. See Niederreiter (1978) for a comprehensive review of the associated theory.

\section{Quasirandom Points}

Suppose one wants to estimate the multivariable integral

$$
\theta=\int_{0}^{1} \ldots \int_{0}^{1} f\left(x_{1}, \ldots, x_{N}\right) d x_{1} \ldots d x_{N}
$$

by the summation

$$
\theta_{K}=\frac{1}{K} \sum_{j=1}^{K} f\left(u_{1 j}, \ldots, u_{N j}\right)
$$

where $\left\{u_{1 j}, \ldots, u_{N j}: j=1, \ldots, K\right\}$ is a sequence of points uniformily distributed in the $N$-dimensional unit hypercube $2_{N}=[0,1]^{N}$. Let $A(R ; K)$ denote the number of soints ${\underset{\sim}{u}}_{j}=\left(u_{1 j}, \ldots, u_{N j}\right) j=1, \ldots, k$ that fall in the region $R \subseteq Q_{N}$. Then ${\underset{\sim}{u}}_{j}, \ldots, u_{K}$ are said to be uniformly distributed in ${ }_{4}$ if

$$
\lim _{K \rightarrow \infty} \frac{A(R ; K)}{K \lambda(R)}=1
$$

for all $R=\left\{\left(x_{1}, \ldots, x_{N}\right): \alpha_{i} \leq x_{i} \leq \beta_{j} ; i=1, \ldots, N\right\}$. Here $\lambda(R)$ is the 
measure of volume of $R$ in $2_{N}$. We define the extreme discrepancy of $u={\underset{\sim}{u}}_{j}, \ldots, u_{k}$ as

$$
D_{K}=D_{K}(N, u)=\sup _{K \subseteq \mathscr{Q}_{N}}\left|\frac{A(R ; K)}{K}-\lambda(R)\right|
$$

for $R=\left\{\left(x_{1}, \ldots, x_{N}\right): 0 \leq x_{i} \leq \beta_{i} ; i=1, \ldots, N\right\}$. If $f$ is of bounded variation in the sense of Hardy and Krause, then

$$
\left|\theta_{K}-\theta\right|<V(f, N) D_{K}
$$

where $V(f, N)$ is a function of the bounded variation of $f$ in $N$ and lower dimensions. Most importantly, uniform sequences exist for which

$$
D_{K} \leq O\left((\log K)^{N / K)}\right. \text {. }
$$

This is an impressive result, for in the special case in which $\left\{u_{i j}: i=1, \ldots, N ; j=1, \ldots, K\right\}$ is a sequence of $i, i, d$. random variables uniformly distributed on $[0,1)$, one has only

$$
\lim _{K \rightarrow \infty} \sup \frac{\sqrt{2 K} D_{K}}{\sqrt{\log \log K}}=1 \quad \text { w.p. 1, }
$$

a result due to Chung (1949) for $N=1$ and to Kiefer (1961) for $N>1$. Halton (1960) describes a uniformly distributed sequence for which (12) holds. If $R \geq 2$ is an integer, then every non-negative integer $n$ has an expansion of the form

$$
\begin{array}{ll}
n=\sum_{i=0}^{m} a_{i} R^{i} & a_{i} \in\{0,1, \ldots, R-1\} \\
& 0 \leq i \leq m \text { and } m=\left\lfloor\log _{R} n\right\rfloor .
\end{array}
$$

Corresponding to this expansion one has the radical inverse function

$$
\phi_{R}(n)=\sum_{i=0}^{m} a_{i} R^{-i-1}
$$

Then Halton shows that the sequence $\left\{\phi_{R_{1}}(j), \ldots, \phi_{R_{N}}(j) ; j=0,1, \ldots, K-1\right\}$, where $R_{1}, \ldots, R_{N}$ are pairwise relatively prime, gives the bound (12). 
We now specialize these results to the network analys is at hand.

Define for $j=1, \ldots, k$

$$
\begin{array}{ll}
x_{i j}=G_{i}\left(u_{i j}\right) & i=1, \ldots, N \\
T_{m j}=\sum_{i=1}^{N} a_{i m} X_{i j} & m=1, \ldots, L \\
Y_{i j}=\sup _{\substack{m=1, \ldots, L \\
a_{i m}=1}} T_{m j}-x_{i_{m} j} & i \in H .
\end{array}
$$

Then it is not difficult to see that. can write $g_{m}$ as a $(\mathrm{N}-|\mathrm{H}|+\mathrm{l})$-dimensional integral over $(\mathrm{H} \mid+1$ with integrand of bounded variation and that (8), using (16), ( d (18), is an approximation to this integral. As a result, one has for a given network using the Halton sequence the deterministic bound

$$
\left|\hat{g}_{m K}-g_{m}\right| \leq O\left((\log K)^{N-|H|+1} / K\right),
$$

which makes clear the desirability of choosing as $H$ the cutset of maximal cardinality.

4. Estimating $\left\{r_{i}\right\},\left\{h_{m}\right\}$ and $\left\{s_{i}\right\}$

This section describes estimators analogous to (8) for

$r_{i}=\operatorname{pr}$ (arc $i$ is on the longest path) $i=1, \ldots, N$

$$
n_{m}=p r(m \text { is the shortest path }) \quad m=1, \ldots, L
$$

and

$$
s_{i}=\operatorname{pr}(\operatorname{arc} i \text { is on the shortest path) } i=1, \ldots, N \text {. }
$$

Before doing so, we explain the motivation for studying $\left\{r_{i}\right\}$ and $\left\{s_{i}\right\}$. If, say, $r_{i}$ turns out to be considerably greater than the remaining arc probabilities on the longest path, then there is good reason to believe that the completion time associated with arc $i$ is a principal determinant 
of the longest path time. This knowledge of the relative importance of arc $i$ may lead to a modification of the activity associated with this arc for the purpose of shortening the longest path time. Analogous considerations apply to $\left\{s_{i}\right\}$. Since

$$
r_{i}=\sum_{m=1}^{L} a_{i m} g_{m}
$$

one has the estimator

$$
\hat{r}_{i K}=\sum_{m=1}^{L} a_{i m} \hat{g}_{m K}
$$

Moreover, it is easily seen that $\left.\left|\hat{r}_{i K}-r_{i}\right| \leq 0(\log K)^{N-\mid H_{i}+1} / K\right)$ for the Halton sequence.

The estimation of $\left\{h_{m}\right\}$ follows closely that for $\left\{g_{m} ;\right.$. Let

$$
\begin{aligned}
& z_{i}= \inf S_{m} \\
& m=1, \ldots, L \\
& a_{i m}=i
\end{aligned} \quad i \in H
$$

so that $x_{i}+z_{i}$ gives the minimal tim of all paths that use arc $i$. il. Then

$$
\begin{aligned}
& \operatorname{pr}\left(m \text { is the shortest path } \mid S_{Y}, \ldots, S_{L}\right) \\
& =\int_{0}^{\infty}\left\{\pi_{\substack{i \in H \\
i \neq i_{m}}}\left[1-F_{i}\left(t-z_{i}\right)\right]\right\} d F_{i_{m}}\left(t-S_{m}\right) \quad \text { if } z_{i}=S_{m i i} \\
& 0
\end{aligned}
$$

Again, let the subscript $j$ denote replication $i$. Also let

$$
B_{m j}=\operatorname{ll}_{\substack{i \in H \\ i \neq i_{m}}}\left[1-F_{i}\left(T_{m j}-Z_{i j}\right)\right] \quad \text { if } \quad T_{m, j}=Z_{i_{m} j}+x_{i m} j
$$

$$
=0 \quad \text { otherwise. }
$$


Then

$$
\hat{h}_{m K}=\frac{1}{K} \sum_{j=1}^{K} \delta\left(T_{m j}-z_{i_{m} j}-x_{i_{m} j}\right) B_{m j}
$$

is our estimator of $h_{m}$ and again for the Halton sequence one has $\left|\hat{h}_{m K}-h_{m}\right| \leq 0\left((\log K)^{N-|H|+1} / K\right\rangle$.

For $\left\{s_{i} ; i=1, \ldots, N\right\}$ note that

$$
s_{i}=\sum_{m=1}^{L} a_{i m} h_{m}
$$

so that our estimator has the form

$$
\hat{s}_{i K}=\sum_{m=1}^{L} a_{i m} \hat{h}_{m K}
$$

with error $\left|\hat{s}_{i K^{-S}}\right| \leq O\left((\log K)^{N-|H|+1} / K\right)$ for the Halton sequence.

\section{Series Reduction}

As shown in Section 2, the rate of convergence of the error of approximation $(10)$ is bounded by a function $0\left((\log K)^{N} / K\right)$. Using the cutset $H$ enables one to bound this convergence more tightly by $O\left((\log K)^{N-|H|+1} / K\right)$ and, presumably, using the cutset $H$ with maximal cardinality is best. In fact, any preanalysis that leads to a smaller exponent for $\log K$ is desirable from the viewpoint of a tighter error bound. The technique of series reduction is one such approach to further reduction.

Figure 1 shows an activity network due to Rattersby (1970) for the partial overhaul of a unit in an oil refinery. Table 1 shows the corresponding incidence matrix. Note that arcs 17 and 18 are both on all eight paths.

Insert Fig. 1 and Table 1 about here.

Therefore, we can ignore these arcs and reduce the number of relevant arcs for analysis from 18 to $N=16$ arcs without any effect on our results. 
Observe that $e_{2}, e_{3}, e_{5}, e_{6}, e_{9}, e_{10}$ and $e_{12}$ form a cutset $H$ for the network. Since $N=16$ arcs and $|H|=7$ the exponent for $\log K$ is

$\mathrm{N}-|H|+\mid=10$. Now note that arcs $e_{1}$ and $e_{4}$ occur only on paths 1 and $2, e_{6}$ and $e_{15}$ occur only on path $5, e_{7}$ and $e_{16}$ occur only on paths 6,7 and 8 and $e_{8}$ and $e_{11}$ occur only on paths 7 and 8 . Suppose we now replace these arc pairs by single arcs $e_{1}+e_{4}, e_{6}+e_{15}$, $e_{7}+e_{16}$ and $e_{8}+e_{11}$. Then the network has $N^{\prime}=12$ arcs and the cutset $H^{\prime}$ with $e_{2}, e_{3}, e_{5}, e_{6}+e_{15}, e_{9}, e_{10}$ and $e_{12}$ has cardinality 7 so that $N^{\prime}-\left|H^{\prime}\right|+\mid=6$ is the resulting exponent for $\log K$ in the bound on $\left|\hat{g}_{m K}-g_{m}\right|$ and $\left|\hat{h}_{m K}-h_{m}\right|$. Note that if one adopts this series reduction it is not possible to estimate $r_{i}$ and $s_{i}$ for those original arcs in the reduction.

The feasibility of implementing this series reduction in practice depends on whether or not the d.fs. of the summed arc times are convenient for computation and on the relative computation costs with and without series reduction. Suppose $W_{1}, \ldots, W_{n}$ are independent exponentially distributed random variables with distinct rates $\beta_{1}, \ldots, \beta_{n}$. Then for $n>1 \quad v_{n}=w_{1}+\ldots+w_{n}$ has the d.f.

$$
F_{V_{n}}(t)=1-\sum_{i=1}^{n} \prod_{\substack{j=1 \\ j \neq i}}^{n}\left(i \beta_{j} / \beta_{j}\right)^{-1} e^{-\beta_{i} t} .
$$

For summed arcs in the cutset one need only replace their individual d.fs. in (8) and (26) by the corresponding d.f. from (29). For summed arcs not in the cutset one needs to solve the equation

$$
v=F_{V_{n}}\left(T^{\prime}\right)
$$


for $T^{\prime}, v$ being the appropriate element of the Halton sequence. This can be done readily by the Newton-Raphson method. A suggested starting point for the iteration is

$$
T_{0}^{\prime}=-[\ln (1-v)] \sum_{i=1}^{n} \frac{1}{\beta_{i}}
$$

We evaluate this approach in our example where we study the relative benefits of the cutset information, quasirandom points and series reduction. As we show, the relative benefit of series reduction becomes more apparent as the sample size $K$ increases. In fact, for small $K$ series reduction may be less preferable.

6. Experimental Design

This section describes the layout for an experiment designed to determine the extent to which quasirandom points lead to accelerated accuracy when estimating $\left\{g_{m}\right\}$ and $\left\{h_{m}\right\}$ for the network in Fig. 1 . In particular, we introduce a degree of randomness into the experiment in order to compute estimates of the mean-square errors of our point estimates. We then study the behavior of these sample mean-square errors as $K$ increases. Note that we choose this method of evaluating our proposed method merely because it is necessary to find a common basis for comparing results based on a deterministic method with baseline results using random sampling.

Consider an experiment consisting of $?$ statistically independent blocks or macroreplications each of $K$ microreplications. Let $i v_{i m}$ : $i=1, \ldots, N ; m=1, \ldots, Q\}$ denote a sequence of $i . i . d$. random variables from $u[0,1)$ and define

$$
c_{i m}=\left(c: \phi_{R_{i}}(c)=v_{i m}\right) \quad i=1, \ldots, N \quad m=1, \ldots, 0 .
$$


Then on macroreplication $m$ we use the quasirandom point $\phi_{R_{1}}\left(C_{1 m}+j-1\right), \ldots, \phi_{R_{N}}\left(C_{N m}+j-1\right)$ on microreplication $j$ for $j=1, \ldots, K$. Let $\hat{\theta}_{j m}$ denote an estimate of a particular quantity $\theta$ computed on microreplication $j$ on macroreplication $m$. Then $\bar{\theta}_{1}, \ldots, \bar{\theta}_{Q}$, where

$$
\bar{\theta}_{m}=\frac{1}{k} \sum_{j=1}^{k} \hat{\theta}_{j m} \quad m=1, \ldots, Q,
$$

are i.i.d. random variables with sample mean-square error

$$
s_{K}^{2}=\frac{1}{Q-1} \sum_{m=1}^{Q}\left(\bar{\theta}_{m}-\overline{\bar{\theta}}_{Q}\right)^{2}
$$

where

$$
\overline{\bar{\theta}}_{Q}=\frac{1}{Q} \sum_{m=1}^{Q} \bar{\theta}_{m}
$$

It is the behavior of $s_{K}^{2}$ versus $K$ that interests us. Note again that the only reason for randomizing the seeds $\left\{v_{i m} ; i=1, \ldots, N\right\}$ on each of the $Q$ macroreplications is to enable us to derive the estimates of mean-square error.

To provide an instructive normalization we also run $M$ statistically independent macroreplications each of $k=1$ microreplication. Let $\hat{\theta}_{1}, \ldots, \hat{\theta}_{M}$ denote the resulting estimates of $\theta$ each with sample mean-square error

$$
w_{M}^{2}=\frac{1}{M-1} \sum_{m=1}^{M}\left(\hat{\theta}_{m}-\tilde{\theta}_{M}\right)^{2}
$$

where

$$
\tilde{\theta}_{M}=\frac{1}{M} \sum_{m=1}^{M} \hat{\theta}_{m} .
$$

Then the quantity $w_{M}^{2} / K s_{K}^{2}$ should increase as $K$ increases if accelerated convergence is occurring. 


\section{Example}

To illustrate the proposed procedures we use the network of Fig. 1 for which we estimate $\left\{g_{m} ; m=1, \ldots, 8\right\}$ and $\left\{h_{m} ; m=1, \ldots, 8\right\}$. Monte Carlo experiments were performed using quasirandom points and the cutset $H^{\prime}=\left\{e_{2}, e_{3}, e_{5}, e_{6}+e_{15}, e_{9}, e_{10}, e_{12}\right\}$ with

Plan A

No series reduction on the remaining arcs $\left\{e_{1}, e_{4}, e_{7}, e_{8}, e_{17}, e_{13}, e_{14}, e_{16}\right\}$

and then with

\section{Plan B}

Series reduction on the remaining arcs

$$
\left\{e_{1}+e_{4}, e_{7}+e_{16}, e_{8}+e_{11}, e_{13}, e_{14}\right\}
$$

Motivation for this choice of plans arose from the observation that plan A requires (29) to be evaluated once per microreplication whereas plan $B$ additionally requires evaluation of (29) and its derivative iteratively (by the Newton-Raphson method) for each of the three reductions $e_{1}+e_{4}, e_{7}+e_{16}$ and $e_{8}+e_{11}$. While it is true that plan $B$ also requires three less quasirandom points per microreplication, the relative cost for an iterative computation needs to be considered explicitly.

Expression (8) and (26) were used to estimate each $g_{m}$ and $h_{m}$ respectively. For the baseline case of pure random sampling $g_{m}$ and $h_{m}$ were estimated by

$$
\tilde{g}_{m M}=\frac{1}{M} \sum_{j=1}^{M} \delta\left(T_{j}^{\star}-T_{m j}\right)
$$

and

$$
\tilde{n}_{m M}=\frac{1}{M} \sum_{j=1}^{M} \delta\left(T_{\star j}-T_{m j}\right),
$$


respectively, where

$$
T_{\star j}=\min \left(T_{1 j}, \ldots, T_{L j}\right)
$$

and

$$
T_{j}^{\star}=\max \left(T_{1 j}, \ldots, T_{L j}\right)
$$

Note that estimates of $\left\{r_{i}\right\}$ and $\left\{s_{i}\right\}$ could in principle also be computed at small marginal cost.

Let $w_{M}^{2}(\rho) / K$ and $s_{K}^{2}(\rho)$ denote the sample mean-square errors for the estimators of $\rho$. Then Table 2 lists for selected $k$ the averages

$$
\beta_{h}(W)=\frac{1}{8} \sum_{m=1}^{8}\left[w_{M}^{2}\left(h_{m}\right) / K s_{K}^{2}\left(h_{m}\right)\right]
$$

for the shortest path using sampling plans $W=A$ and $W=B$

Insert Table 2 about here.

in columns 3 and 4 , respectively, and the averages

$$
B_{g}(W)=\frac{1}{8} \sum_{m=1}^{8}\left[w_{M}^{2}\left(g_{m}\right) / K s_{K}^{2}\left(g_{m}\right)\right]
$$

for the longest path in columns 5 and 6 respectively.

These results are most encouraging for they indicate substantive increases in these ratios as the number of microreplications $k$ increases. Also, they show the relative desirability of using series reduction as $K$ increases. Note that computation time considerations limited the number of macroreplications for $k>2^{11}$.

When evaluating a proposed accuracy accelerating technique one also neefs to study its cost relative to the cost of crude Monte Carlo sampling. One way to make this assessment is in terms of the CPU times required per observation with each sampling plan. To simplify the discussion, 
let us concentrate on the estimation of, say, $g_{m}$. Suppose that a single observation using pure random sampling has mean-square error $V$. Then over $k_{1}$ independent observations or microreplications the resulting estimate of $g_{m}$ has mean-square error $V_{K_{1}}=V / K_{1}$. Suppose that for $k$ observations of the quasirandom point approach the estimator of $g_{m}$ has mean-square error $\Omega_{K}$. Let $\omega_{W}$ denote the ratio of cost per microreplication for plan $W$ using quasirandom points over the cost per microreplication using independent random sampling. If we begin by taking $K$ observations using plan $A$ and $K_{1}=\omega_{A} K$ observations using independent random sampling, then each experiment takes exactly the same amount of time. Moreover, the ratio of their mean-square errors is

$$
\frac{V_{K}}{\Omega_{K}}=\frac{V / K_{1}}{\Omega_{K}}=\frac{1}{{ }^{\omega_{A}}} \cdot \frac{V / K}{\Omega_{K}} \text {. }
$$

Let $\hat{\theta}_{i}$ denote the value of the estimate on independent observation $i=1, \ldots, M$. To estimate $V$ we use $w_{M}^{2}$ so that $w_{M}^{2} / K$ is the estimate of mean-square error $V / K$ for the case of pure random sampling for any $K$. Also $\left\{s_{K}^{2}: k=2^{j} ; j=7, \ldots, 14\right\}$ are the estimates of mean-square errors $\left\{\Omega_{K}\right\}$ for plan $A$ for the selected values of $K$.

To estimate the average ratios of mean-square errors for equal run times (using $K_{1}=\omega_{W} K$ ) we use $B_{h}(W) / \omega_{W}$ and $B_{g}(W) / \omega_{W}$ for sampling plans $W=A, B$. For the case of pure random sampling one microreplication required an average of 7.37 microseconds on an IBM $4341 / M 2$ whereas plan A required an average of 12.25 microseconds per microreplication. Plan $B$ required an average of 14.85 microseconds per microreplication. Therefore, $1 / \omega_{A}=7.37 / 12.25=.50$ when comparing random sampling to plan $A$ and $1 / \omega_{B}=7.37 / 14.85=.49$ when comparing random sampling to 
plan B. Columns 7 through 10 show $B_{h}(A) / \omega_{A}, B_{h}(B) / \omega_{B}, B_{g}(A) / \omega_{A}$ and $B_{g}(B) / \omega_{B}$, respectively for selected numbers of microreplications $K$.

These results are sobering for they show that the relative desirabiiity of series reduction in the noncutset arcs does not set in until $k=2^{11}$ for $\left\{h_{m}\right\}$ and for $\left\{g_{m}\right\}$. Let us now put this cost analysis into perspective. Since $\omega_{A}$ and $\omega_{B}$ are essentially independent of $k$ and gene-ally $\omega_{B}>\omega_{A}$, there is always a crossover point $K^{*}$ for each network at which one prefers plan $A$ to plan $B$ whenever $K<K^{\star}$ and prefers plan B to plan $A$ whenever $K>K^{*}$. Since for an arbitrary network one does not know $K^{\star}$, the choice between the two plans is not trivial. Therefore, the advice to do series reduction whenever possible cannot be accepted unqualifiedly unless the planned number of microreplications $k$ is substantially large. 


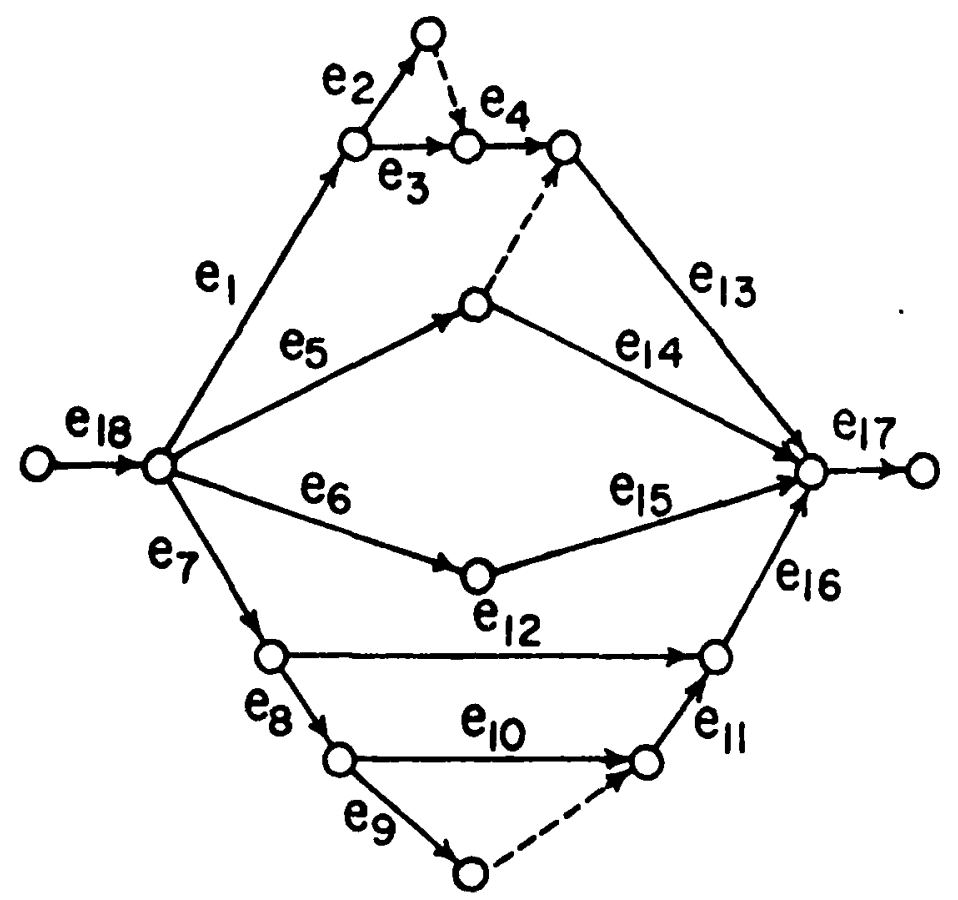

Figure 1. Battersby Network 


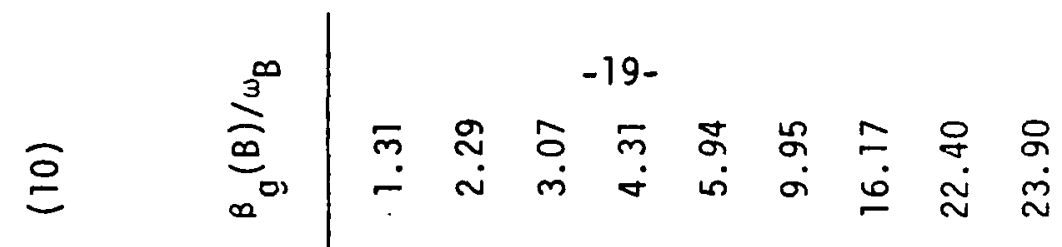

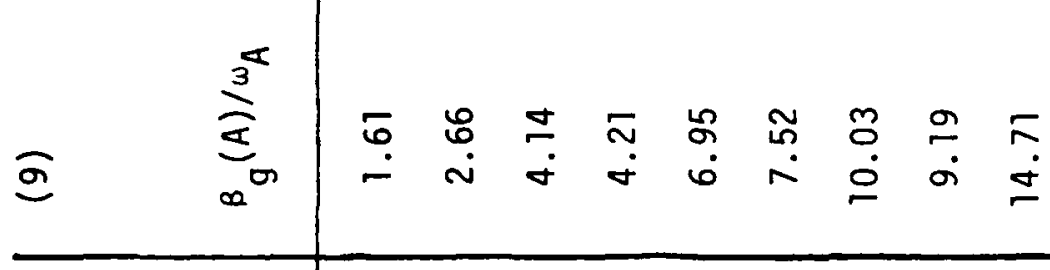

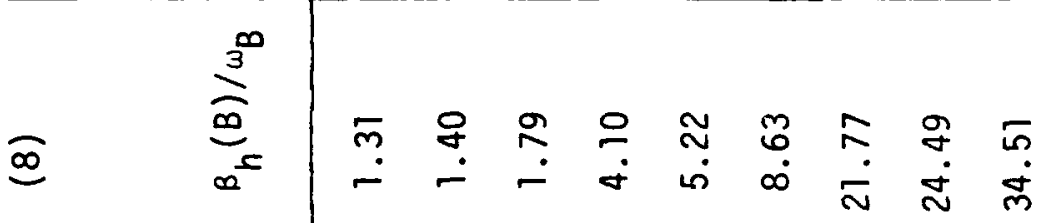

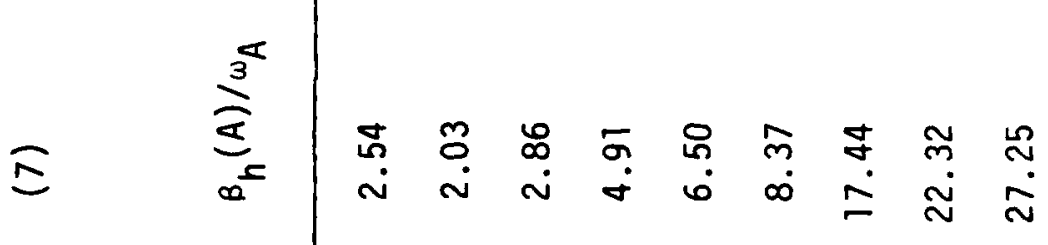

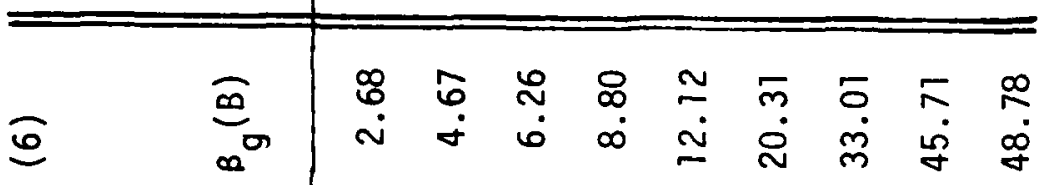

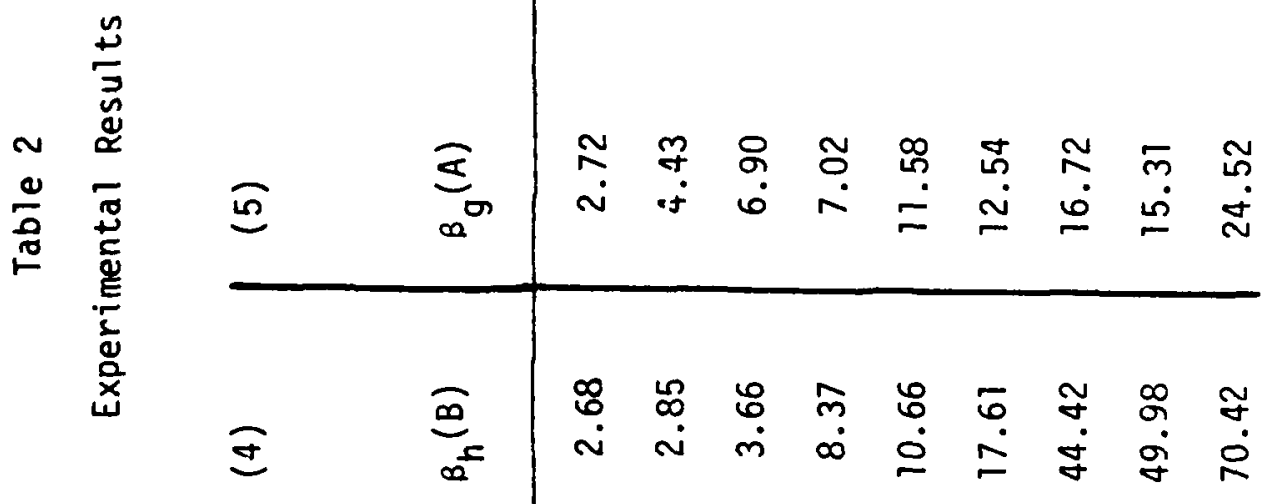

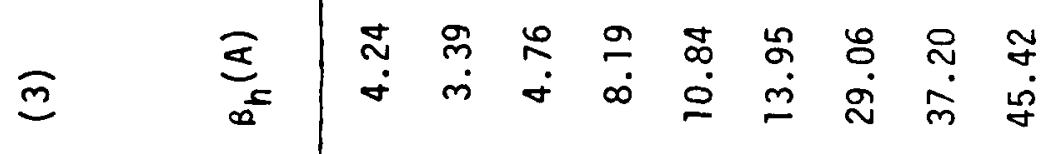

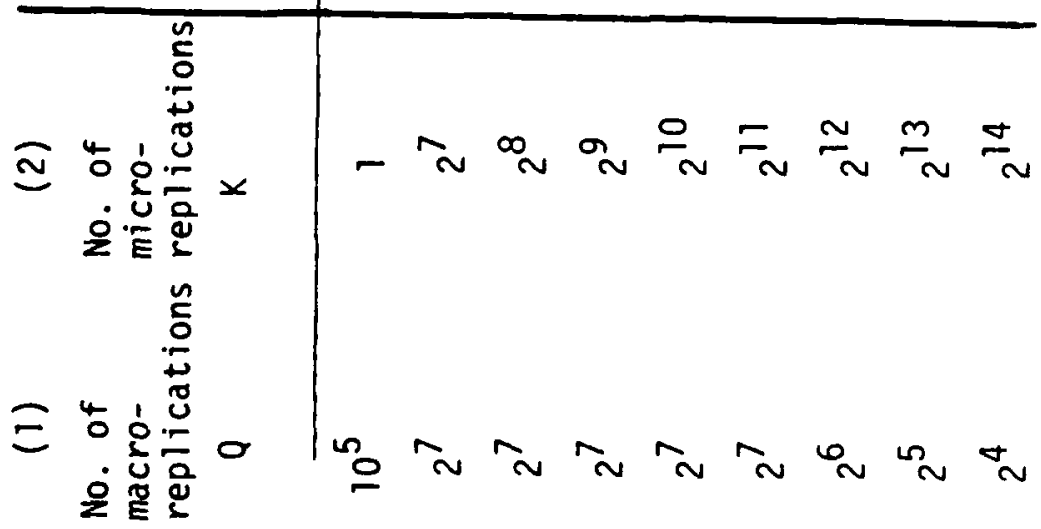




\section{References}

1. Battersby, A. (1970). Network Analys is for Planning and Scheduling, John Wiley and Sons, third edition.

2. Chung, K.L. (1949). "An Estimate Concerning the Kolmogoroff Limit Theorem," Trans. Amer. Math. Soc., 67, 36-40.

3. Elmaghraby, S.E. (1977). Activity Networks: Project Planning and Control by Network Models, John Wiley and Sons.

4. Fishman, G.S. (1983). "Variance Reduction in the Simulation of Stochastic Activity Networks," TR 83/2, Curriculum in Operations Research and Systems Analysis, University of North Carolina at Chapel Hill.

5. Halton, J.H. (1960). "On the Efficiency of Certain Quasirandom Sequences of Points in Evaluating Multi-dimensional Integrals," Numerische Mathematik, 2, 84-90.

6. Kiefer, J. (1961). "On Large Deviations of the Empiric d.f. of Vector Chance Variables and a Law of the Iterated Logarithm," Pacific J. Math., 11, 649-660.

7. Niederreiter, H. (1978). "Quasi-Monte Carlo Methods and Pseudo-random Numbers," Bul1. Amer. Math. Soc., 84, 957-1041.

8. Sigal, C.C., A.A.B. Pritsker and J.J. Solberg (1979). "The Use of Cutsets in Monte Carlo Analysis of Stochastic Networks," Mathematics and Computers in Simulation, $21,376-384$.

9. Sullivan, R.S., J.C. Hayya and R. Schaul (1982). "Efficiency of the Antithetic Variate Method for Simulating Stochastic Networks," Management Science, 28, 563-572. 
INCLASSIEIED

SECU:ITY CLASSIFICATION OF THIS PAGE (When Defa Kntered)

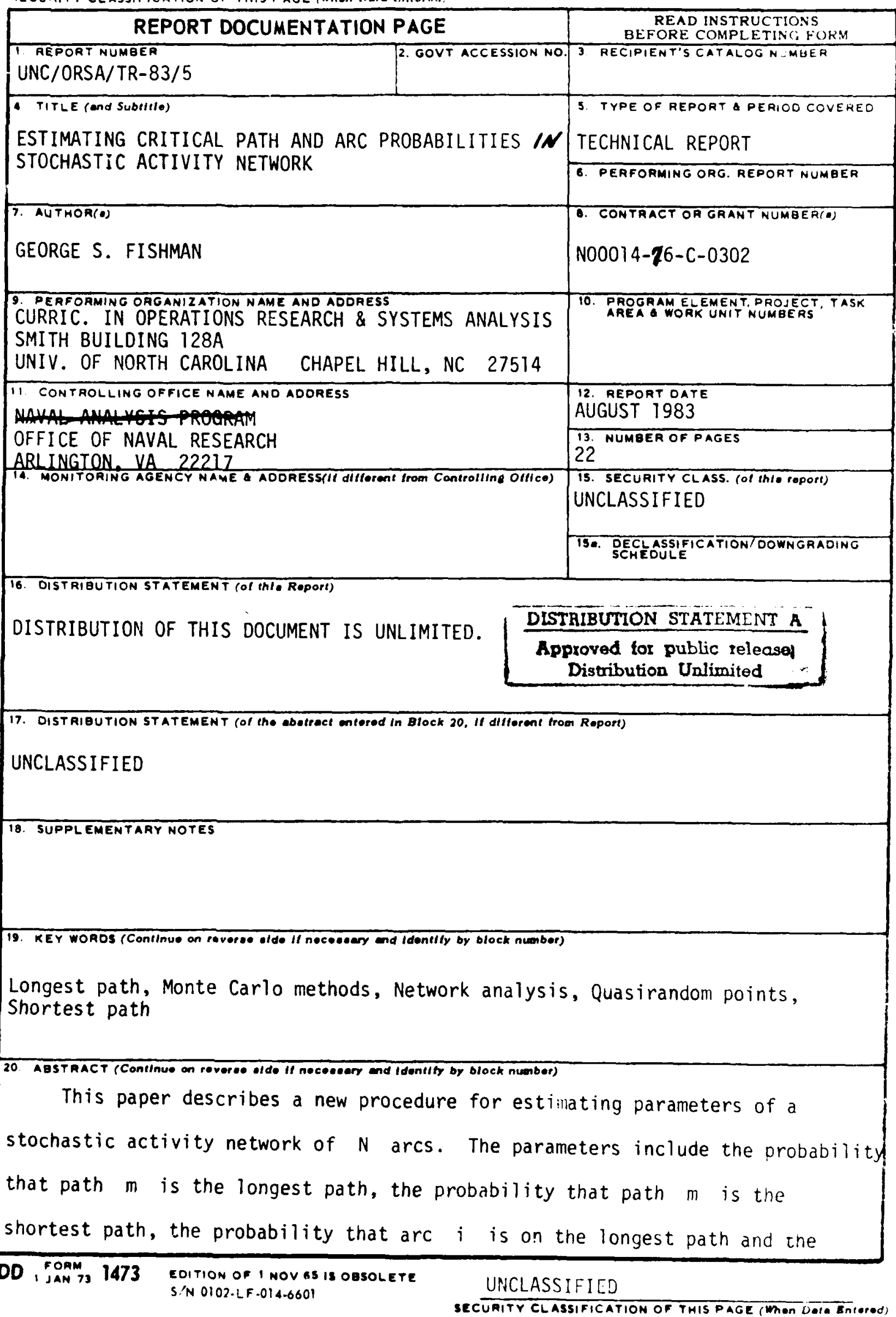


probability that arc $i$ is on the shortest path. The proposed procedure uses quasirandom points together with information on a cutset $H$ of the network to produce an upper bound of $O\left((\log K)^{N-|H|+1} / K\right)$ on the absolute error of approximation where $K$ denotes the number of replications. This is a deterministic bound and is more favorable than the convergence rate of $1 / k^{1 / 2}$ that one obtains for the standard error for $k$ independent replications using random sampling. It is also shown how series reduction can improve the convergence rate by reducing the exponent on $\log k$. The technique is illustrated using a Monte Carlo sampling experiment for a rietwork of 16 relevant arcs with a cutset of $H=7$ arcs. The illustration shoris the superior performance of using quasirandom points with a cutset (plan A) and the even better performance of using quasirandom points with the cutset together with series reduction ( $p l a n B$ ) with regard to mean-square error. However, it also shows that computation time considerations favor plan $A$ when $K$ is small and plan $B$ when $K$ is large. 